\title{
Elastic Properties of Double-Layered Manganite $\mathrm{La}_{1.2} \mathrm{Sr}_{1.8-x} \mathrm{Ba}_{x} \mathrm{Mn}_{2} \mathrm{O}_{7}$ $(x=0.0-0.4)$
}

\author{
Y.S. Reddy ${ }^{a, b}$ V. PRAShanth KumaR ${ }^{a}$, S. RAmesh ${ }^{a}$, \\ S. Venkanna ${ }^{a}$, M.V. Ramana Reddy ${ }^{a}$, P. Kistaiah ${ }^{a}$ \\ AND C. VishnUVARDHAN REDDY ${ }^{a, *}$ \\ ${ }^{a}$ Department of Physics, Osmania University \\ Hyderabad, 500 007, India \\ ${ }^{b}$ Chitanya Bharathi Institute of Technology \\ Gandipet, Hyderabad, 500 075, India
}

(Received November 5, 200\%; in final form December 19, 2007)

The elastic behavior of the sol-gel prepared double-layered manganite system $\mathrm{La}_{1.2} \mathrm{Sr}_{1.8-x} \mathrm{Ba}_{x} \mathrm{Mn}_{2} \mathrm{O}_{7}(x=0.0-0.4)$ was investigated at $300 \mathrm{~K}$, employing ultrasonic pulse transmission technique at $1 \mathrm{MHz}$. The values of elastic moduli and acoustic Debye temperature $\left(\theta_{\mathrm{D}}\right)$ were computed from longitudinal and shear velocities. The measured values were corrected to zero porosity using Hasselman and Fulrath's formula. The elastic constants of the samples, estimated using Modi's heterogeneous metal-mixture rule, were also reported. The variation of elastic moduli with $\mathrm{Ba}$ concentration was interpreted in terms of strength of interatomic bonding.

PACS numbers: 75.47.Gk, 75.47.Lx

\section{Introduction}

The double-layered manganites with general compositional formula $\mathrm{La}_{2-2 x} \mathrm{Sr}_{1+2 x} \mathrm{Mn}_{2} \mathrm{O}_{7}$ have been studied extensively due to their colossal magnetoresistance (CMR) at moderate magnetic fields [1, 2]. Particularly, $\mathrm{La}_{1.2} \mathrm{Sr}_{1.8} \mathrm{Mn}_{2} \mathrm{O}_{7}$ compound with $\mathrm{Ca}^{2+}$ and $\mathrm{Ba}^{2+}$ substitution for $\mathrm{Sr}^{2+}$ has been investigated with respect to its magnetotransport properties [3-6]. To learn about the suitability of a specific material into functional device applications, general idea of mechanical properties is essential. As the ceramic CMR materials are subjected to magnetic fields, large stresses will develop inside the material and therefore it is always desirable to look into the mechanical properties of these materials. Hence,

*corresponding author; e-mail: reddycvv@osmania.ac.in 
the determination of the elastic constants of these materials will provide ample information regarding mechanical strength, fracture toughness, and thermal shock resistance. In view of this, a systematic study has been undertaken to evaluate the elastic moduli of $\mathrm{Ca}^{2+}$ and $\mathrm{Ba}^{2+}$ doped $\mathrm{La}_{1.2} \mathrm{Sr}_{1.8} \mathrm{Mn}_{2} \mathrm{O}_{7}$ system employing ultrasonic pulse-transmission (UPT) technique at room temperature [7]. In this paper, we report the values of the elastic constants of $\mathrm{La}_{1.2} \mathrm{Sr}_{1.8}{ }_{x} \mathrm{Ba}_{x} \mathrm{Mn}_{2} \mathrm{O}_{7}$ $(x=0.0-0.4)$ system.

\section{Experimental}

The $\mathrm{La}_{1.2} \mathrm{Sr}_{1.8-x} \mathrm{Ba}_{x} \mathrm{Mn}_{2} \mathrm{O}_{7}(x=0.0-0.4)$ powders have been prepared by the sol-gel method [8]. High pure powders of $\mathrm{La}_{2} \mathrm{O}_{3}, \mathrm{MnCO}_{3}, \operatorname{Sr}\left(\mathrm{NO}_{3}\right)_{2}$ and $\mathrm{Ba}\left(\mathrm{NO}_{3}\right)_{2}$ weighted in appropriate proportions were used to obtain the nominal compositions of $\mathrm{La}_{1.2} \mathrm{Sr}_{1.8-x} \mathrm{Ba}_{x} \mathrm{Mn}_{2} \mathrm{O}_{7}(x=0.0-0.4) . \mathrm{La}_{2} \mathrm{O}_{3}$ and $\mathrm{MnCO}_{3}$ were converted into nitrates prior to use. All the nitrates were dissolved in the citric acid solution and then the $\mathrm{pH}$ was adjusted to $\approx 6$ with ammonia solution. After getting the water evaporated, ethylene glycol was added and heated at about $90^{\circ} \mathrm{C}$ until a gel-type solution is formed. The gel was dried at $150^{\circ} \mathrm{C}$ and then decomposed at $250^{\circ} \mathrm{C}$ in air for $2 \mathrm{~h}$ to decompose nitrates and all organic materials. The resultant ash was ground to get a fine homogeneous powder. The powder was calcinated in air at $1100^{\circ} \mathrm{C}$ for $10 \mathrm{~h}$ and then pressed into circular pellets. The pellets were finally sintered in air at $1400^{\circ} \mathrm{C}$ for $6 \mathrm{~h}$.

The structural characterization was carried out by X-ray diffraction (XRD) using $\mathrm{Cu} \mathrm{K}_{\alpha}$ radiation. Bulk densities of the samples were determined by the Archimedes principle using xylene as buoyant to get fairly good results. The X-ray densities $\left(\rho_{x}\right)$ and porosity fraction were determined by employing the formulae $C=1-\rho / \rho_{x}$.

The ultrasonic measurements were carried out by the UPT technique at room temperature $(300 \mathrm{~K})$ [9]. Longitudinal and shear velocities have been measured using $X$ - and $Y$-cut quartz transducers, respectively, with a fundamental frequency of $1 \mathrm{MHz}$. The rf pulses generated by the pulse oscillator were applied to the transmitting transducer, which converts them into acoustic pulses. These acoustic pulses, after propagating through the test sample, were converted back into electrical signals by the receiving transducers. The amplified output signal was displayed on a $100 \mathrm{MHz}$ digital storage oscilloscope (Tektronix model No. 2221). The difference in time $\Delta T$ between two overlapping received pulse trains was noted using a timer. The velocity of sound was measured using the equation $V=t / \Delta T$, where $t$ is the thickness of the sample. The overall accuracy of these measurements is $\pm 10 \mathrm{~ms}^{-1}$.

\section{Results and discussion}

The XRD patterns of $\mathrm{La}_{1.2} \mathrm{Sr}_{1.8-x} \mathrm{Ba}_{x} \mathrm{Mn}_{2} \mathrm{O}_{7}(x=0.0-0.4)$, shown in Fig. 1, reveal that the samples are of $\mathrm{Sr}_{3} \mathrm{Ti}_{2} \mathrm{O}_{7}$-type structure with a tetragonal unit 
cell (space group: $I 4 / \mathrm{mmm}$ ). It is also observed that the lattice constants ( $a$ and $c$ ) and cell volume $(V)$ increase as $\mathrm{Ba}$ content increases, which is due to the substitution of larger $\mathrm{Ba}^{2+}$ ions (1.47 $\AA$ for coordination number 9$)$ into smaller $\mathrm{Sr}^{2+}(1.31 \AA$ for coordination number 9$)$ sites [10]. The variations of cell volume $(V)$, X-ray density $\left(\rho_{x}\right)$ and bulk density $(\rho)$ with Ba content $(x)$ are depicted in Fig. 2 .

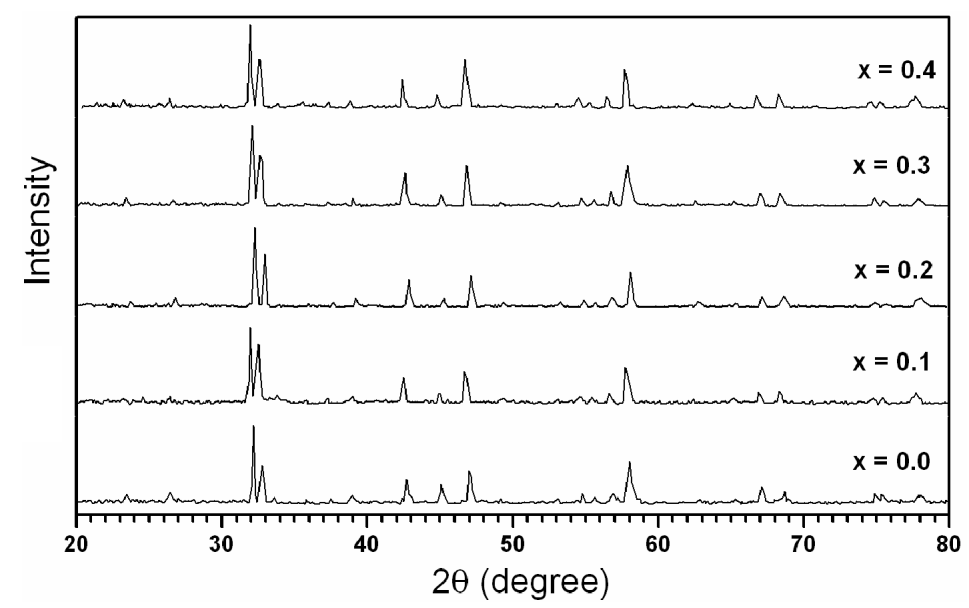

Fig. 1. XRD patterns of $\mathrm{La}_{1.2} \mathrm{Sr}_{1.8-x} \mathrm{Ba}_{x} \mathrm{Mn}_{2} \mathrm{O}_{7}(x=0.0-0.4)$.

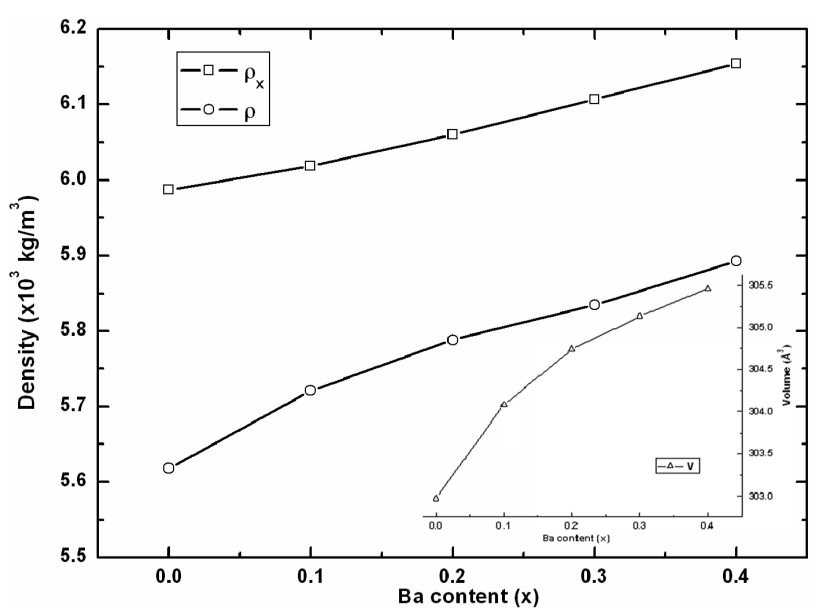

Fig. 2. Variation of X-ray density $\left(\rho_{x}\right)$ and bulk density $(\rho)$ with Ba content $(\mathrm{x})$. Inset figure shows the lattice volume vs. $x$.

The values of elastic constants were calculated using the longitudinal $\left(v_{\mathrm{L}}\right)$ and shear $\left(v_{\mathrm{S}}\right)$ velocities obtained from UPT and employing the following formulae [11]: 
longitudinal modulus $L=\rho v_{\mathrm{L}}^{2}$,

shear modulus $G=\rho v_{\mathrm{S}}^{2}$,

bulk modulus $B=L-\frac{4}{3} G$,

Poisson's ratio $\sigma=\frac{3 B-2 G}{6 B+2 G}$,

Young's modulus $E=(1+\sigma) 2 G$.

The acoustic Debye temperature $\left(\theta_{\mathrm{D}}\right)$, at which nearly all modes of vibrations in a solid are excited, plays an important role in the study of a large number of solid-state problems involving lattice vibrations. Hence, the values of $\theta_{\mathrm{D}}$ were determined using the following Anderson formula [12]:

$$
\theta_{\mathrm{D}}=\frac{h}{k_{\mathrm{B}}}\left(\frac{3 \rho N_{\mathrm{A}} q}{4 \pi M}\right)^{1 / 3} v_{\mathrm{m}},
$$

where $h$ is the Planck constant, $k_{\mathrm{B}}$ is the Boltzmann constant, $\rho$ is the bulk density of the sample, $N_{\mathrm{A}}$ is the Avogadro number, $q$ is the number of atoms in the unit formula (in the present case $q=12$ ), $M$ is the molecular weight and $v_{\mathrm{m}}$ is the mean sound velocity and is given by

$$
v_{m}=\left[\frac{1}{3}\left(\frac{1}{v_{\mathrm{L}}^{3}}+\frac{2}{v_{\mathrm{S}}^{3}}\right)\right]^{-1 / 3} .
$$

The measured values of elastic constants are summarized in Table I. As the materials under the present investigation are porous $(C \approx 0.04-0.06)$, the measured elastic moduli were corrected to zero porosity using the following Hasselman and Fulrath (HF) formulae [13]:

$$
\begin{aligned}
\frac{1}{E_{0}} & =\left[1-\frac{3 C(1-\sigma)(9+5 \sigma)}{2(7-5 \sigma)}\right], \\
\frac{1}{G_{0}} & =\frac{1}{G}\left[1-\frac{15 C(1-\sigma)}{7-5 \sigma}\right], \\
B_{0} & =\frac{E_{0} G_{0}}{3\left(3 G_{0}-E_{0}\right)}, \\
\sigma_{0} & =\frac{E_{0}}{2 G_{0}}-1 .
\end{aligned}
$$

Recently, Modi et al. [14] have developed a model, known as Modi heterogeneous metal-mixture rule (MMMR) to estimate the elastic constants of La-based perovskites. According to this model, the value of elastic constant or acoustic Debye temperature can be calculated by using the following formula: 
TABLE I

Porosity fraction $(C)$, longitudinal velocity $\left(v_{\mathrm{L}}\right)$, shear velocity $\left(v_{\mathrm{S}}\right)$, mean velocity $\left(v_{\mathrm{m}}\right)$, longitudinal modulus $(L)$, shear modulus $(G)$, bulk modulus $(B)$, Young's modulus $(E)$, Poisson's ratio $(\sigma)$ and acoustic Debye temperature $\left(\theta_{\mathrm{D}}\right.$, Fig. 3) for $\mathrm{La}_{1.2} \mathrm{Sr}_{1.8-x} \mathrm{Ba}_{x} \mathrm{Mn}_{2} \mathrm{O}_{7}(x=0.0-0.4)$

\begin{tabular}{c|c|c|c|c|c|c|c|c|c|c}
\hline \hline$x$ & \multirow{2}{*}{$C$} & $v_{\mathrm{L}}$ & $v_{\mathrm{L}}$ & $v_{\mathrm{L}}$ & $L$ & $G$ & $B$ & $E$ & $\sigma$ & \multirow{2}{*}{$\begin{array}{c}\theta_{\mathrm{D}} \\
\end{array}$} \\
\cline { 3 - 8 } & \multicolumn{3}{|c|}{$[\mathrm{m} / \mathrm{s}]$} \\
\hline 0.0 & 0.062 & 3860 & 2245 & 2491 & 83.72 & 28.31 & 45.97 & 70.47 & 0.24 & 311.72 \\
0.1 & 0.049 & 3801 & 2206 & 2448 & 82.67 & 27.85 & 45.54 & 69.40 & 0.24 & 307.34 \\
0.2 & 0.045 & 3743 & 2174 & 2412 & 81.10 & 27.36 & 44.62 & 68.18 & 0.24 & 303.11 \\
0.3 & 0.044 & 3681 & 2141 & 2375 & 79.11 & 26.75 & 43.44 & 66.58 & 0.24 & 298.39 \\
0.4 & 0.042 & 3611 & 2101 & 2331 & 76.88 & 26.02 & 42.18 & 64.75 & 0.24 & 292.93
\end{tabular}

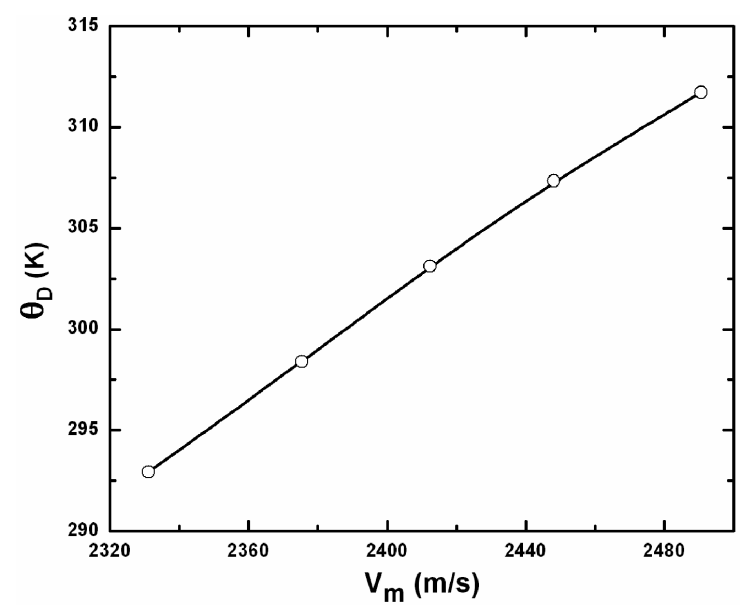

Fig. 3. Variation of acoustic Debye temperature $\left(\theta_{\mathrm{D}}\right)$ with mean sound velocity $\left(v_{\mathrm{m}}\right)$.

$$
K_{\mathrm{pm}}^{\star}=\frac{1}{n} \sum_{i>0, n=1}^{\infty} C_{i n} K_{n},
$$

where $K_{p m}^{*}$ is either elastic modulus or acoustic Debye temperature of the composition to be estimated, $n$ - the total number of metal cations in the unit chemical formula ( $n=5$ in the present case), $c_{i n}$ — the concentration of the n-th cation in the formula unit, while $K_{n}$ is the corresponding modulus or acoustic Debye temperature of the metallic element. The elastic moduli and the Debye temperature values of the metallic elements are taken from the literature $[15,16]$ to estimate $K_{p m}^{\star}$. The values of elastic moduli estimated using MMM rule and obtained from UPT show the similar trend of decrease with Ba doping. However, the values of elastic moduli obtained from UPT show a large variation with Ba content as compared to moderate change predicted by MMM rule (Table II). 
TABLE II

Shear modulus $\left(G_{0}\right)$, bulk modulus $\left(B_{0}\right)$, and Young's modulus $\left(E_{0}\right)$ corrected to zero porosity using $\mathrm{HF}$ and Shear modulus $\left(G^{\star}\right)$, bulk modulus $\left(B^{\star}\right)$ and Young's modulus $\left(E^{\star}\right)$ estimated using MMMR

\begin{tabular}{c|c|c|c|c|c|c|c}
\hline \hline$x$ & $G_{0}$ & $B_{0}$ & $E_{0}$ & \multirow{2}{*}{$\sigma_{0}$} & $G^{\star}$ & $B^{\star}$ & $E^{\star}$ \\
\cline { 2 - 5 } \cline { 5 - 7 } & \multicolumn{3}{|c}{$[\mathrm{GPa}]$} & & \multicolumn{3}{|c}{$[\mathrm{GPa}]$} \\
\hline 0.0 & 32.20 & 53.24 & 80.40 & 0.25 & 36.21 & 47.99 & 77.44 \\
0.1 & 30.84 & 51.18 & 77.05 & 0.25 & 36.17 & 47.89 & 77.23 \\
0.2 & 30.01 & 49.58 & 74.91 & 0.25 & 36.14 & 47.86 & 77.22 \\
0.3 & 29.30 & 48.20 & 73.10 & 0.25 & 36.12 & 47.83 & 77.20 \\
0.4 & 28.38 & 46.55 & 70.76 & 0.25 & 36.10 & 47.80 & 77.19
\end{tabular}

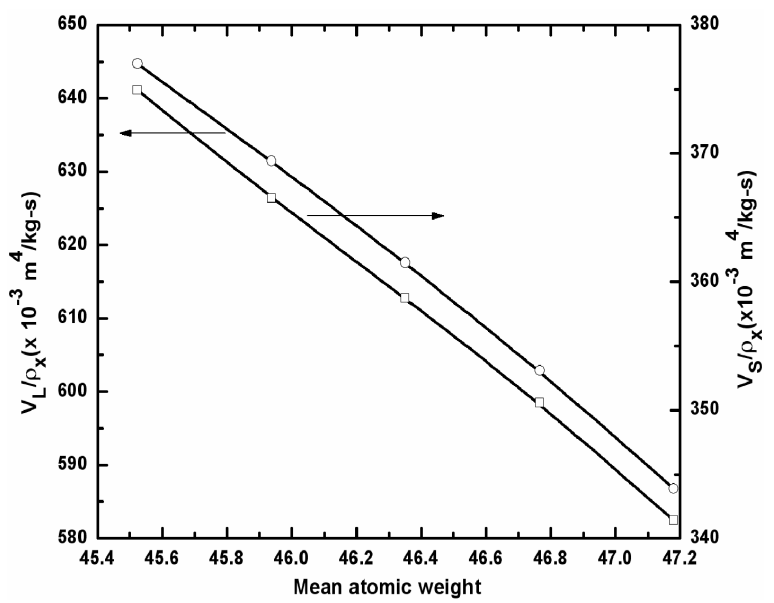

Fig. 4. Variation of $v_{\mathrm{L}} / \rho_{x}$ and $v_{\mathrm{S}} / \rho_{x}$ with mean atomic weight $(M / q)$.

The variation in elastic parameters with Ba content can be interpreted in terms strength of interatomic bonding [17]. When smaller $\mathrm{Sr}^{2+}$ ions (1.31 $\left.\AA\right)$ are replaced by larger $\mathrm{Ba}^{2+}(1.47 \AA)$ ions, the length of interatomic bonding increases and results in the decrease in strength of interatomic bonding, which in turn decreases the elastic moduli. From the above results, it can be observed that both measured and corrected values of elastic moduli change in the same manner and it confirms the quality of the test samples.

The measured and corrected values of Poisson's ratio are found to be in good agreement (Tables I and II) and lie in the theoretical range from -1 to 0.5 . The increasing $\mathrm{Ba}$ concentration results in the decrease in the acoustic Debye temperature (Table I), which suggests the enhancement in lattice vibrations with Ba substitution [14]. The linear relationship between mean sound velocity and acoustic Debye temperature can be seen in Fig. 3 . 
In case of oxides having the same mean atomic weight $(M / q)$, the variation in the mean atomic weight corresponds to the variation in the values of $v_{\mathrm{L}} / \rho_{x}$ and $v_{\mathrm{S}} / \rho_{x}$, because the product of $M / q$ with $v_{\mathrm{L}} / \rho_{x}$ and $v_{\mathrm{S}} / \rho_{x}$ should be a constant $[9,18,19]$. The similar behavior can be seen from Fig. 4, thus establishing the fact that these materials behave like any other oxide materials described in the literature.

\section{Conclusions}

The values of elastic moduli of double-layered manganites $\mathrm{La}_{1.2} \mathrm{Sr}_{1.8-x} \mathrm{Ba}_{x} \mathrm{Mn}_{2} \mathrm{O}_{7} \quad(x=0.0-0.4)$ at room temperature were reported and porosity corrections were also presented. The observed decrease in the elastic constants with Ba substitution suggests the weakening of the interatomic bonding with the increase in Ba content. The decrease in acoustic Debye temperature suggests the enhancement of lattice vibrations with Ba substitution. Further, it was proved that the materials under the present investigation have the similar elastic behavior like other ceramic oxides.

\section{References}

[1] Y. Moritomo, A. Asamitsu, H. Kuwahara, Y. Tokura, Nature (London) 380, 141 (1996).

[2] T. Kimura, Y. Tokura, Ann. Rev. Mater. Sci. 30, 451 (2000).

[3] C.H. Shen, R.S. Liu, S.F. Hu, C.Y. Huang, H.S. Sheu, J. Appl. Phys. 86, 2178 (1999).

[4] P.V. Patanjali, P. Theule, Z. Zhai, N. Hakim, S. Sridhar, R. Suryanarayanan, M. Apostu, G. Dhalenne, A. Revcolevschi, Phys. Rev. B 60, 9286 (1999).

[5] Hong Zhu, Sun Tan, Wei Tong, Yu Heng Zhang, Appl. Phys. Lett. 80, 3778 (1996).

[6] H.K.Singh, K. Ajay Gupta, P.K. Siwach, O.N. Srivastava, J. Magn. Magn. Mater. 292, 483 (2005).

[7] Y.S. Reddy, M.V. Ramana Reddy, P. Veerasomaiah, C. Vishnuvardhan Reddy, Mater. Sci. (Poland) 25, 619 (2007).

[8] G.Ch. Kostogloudis, Vasilakos, Ch. Ftikos, J. Europ. Ceram. Soc. 17, 1513 (1997).

[9] Y.S. Reddy, V. Prashanth Kumar, P. Kistaiah, C. Vishnuvardhan Reddy, J. Alloys Comp. 424, 46 (2006).

[10] R.D. Shannon, Acta Crystallogr. A 32, 751 (1976).

[11] V. Baldev, Raj P. Rajendran, Palanichamy, Science and Technology of Ultrasonics, Narosa Publishing House, New Delhi 2004, p. 250.

[12] Q.L. Anderson, J. Phys. Chem. Solids 24, 909 (1963).

[13] D.P.H. Hasselman, R.M. Fulrath, J. Am. Ceram. Soc. 47, 52 (1964).

[14] K.B. Modi, M.C. Chhantbar, H.H. Joshi, Ceram. Inter. 32, 111 (2006). 
[15] V.Raghavan, Materials Science and Engineering, 5th ed., Prentice-Hall of India Pvt. Ltd., New Delhi 2004, p. 394.

[16] www.webelements.com.

[17] W.A. Wooster, Rep. Prog. Phys. 16, 62 (1953).

[18] O.L. Anderson, in: Physical Acoustics, Vol. 3B, Ed. W.P. Mason, Academic Press, New York 1965, p. 43.

[19] Nitendar Kumar, Y. Purushotham, P. Venugopal Reddy, Z.H. Zaidi, Pran Kishan, J. Magn. Magn. Mater. 192, 116 (1999). 